\title{
Scattering Analysis of Silver Nanoparticles for Solar Cell Applications using Integral Equations
}

\author{
Ismail Enes Uysal \\ Physics Department, Bilkent University, Ankara, Turkey \\ Division of Computer, Electrical and Mathematical Science \\ and Engineering, King Abdullah University of Science and \\ Technology (KAUST), Thuwal, Saudi Arabia \\ Huseyin Arda Ulku \\ Department of Electronics Engineering, Gebze Technical \\ University, Kocaeli, Turkey
}

\author{
Hakan Bagci \\ Division of Computer, Electrical and Mathematical Science \\ and Engineering, King Abdullah University of Science and \\ Technology (KAUST), Thuwal, Saudi Arabia \\ Oguz Gulseren \\ Physics Department, Bilkent University, Ankara, Turkey
}

\begin{abstract}
Plasmonic nanoparticles (NPs) can be used to improve the efficiency of solar cells. Analysis of electromagnetic scattering from NPs is often carried out under the assumptions that they reside in air and have "ideal" shapes (sphere, cube, etc.) However, in a realistic setup, nanoparticles are fabricated on a substrate and their shape and size cannot be controlled precisely. In this work, a surface integral equation solver is used to accurately characterize the scattering from a realistic system, where silver hemispheres of varying sizes are fabricated on an indium tin-oxide substrate. Results obtained by the solver are compared to the experimental results obtained for a similar system.
\end{abstract}

Keywords-Electromagnetic scattering, plasmonics, solar cell, surface integral equations

\section{INTRODUCTION}

The efficiency of solar cells has been increasing steadily in the last two decades however significant improvements are still needed for them [1] to be able to compete with conventional energy sources. One way of increasing the efficiency of solar cells is to employ plasmonic nanoparticles (NPs) [2]. Ideally, NPs increase the efficiency by directing/focusing light energy into the active region of the solar cell. This means that NPs with high scattering cross section (SCS) but low absorption cross section (ACS) have to be designed and fabricated for enhanced efficiency.

SCS of an NP depends on the material, shape, and size as well as background medium it resides in. Scattering from various ideal NP geometries such as sphere, cylinder, cube, and tetrahedron has been analyzed using different numerical methods [2,3]. Oftentimes, in these scattering scenarios, a single ideally-shaped NP resides in an unbounded background medium. However, to accurately predict the output of a real experiment, simulations should take into account multiple nonideally shaped NPs with varying sizes residing on a substrate.

In this work, electromagnetic scattering from a group of silver hemisphere-shaped NPs with varying radii, which are located on a dielectric substrate, is analyzed using a surface integral equation (SIE) solver [4]. Comparison of the SCS computed by the SIE solver to the experimental results demonstrates that simulations, which model multiple NPs with varying sizes and located on a substrate, yield more accurate results than those modeling a single NP that resides in air.

\section{FORMULATION}

The numerical scheme used in this work solves the wellknown Poggio-Miller-Chan-Harrington-Wu-Tsai (PMCHWT) SIE $[4,5]$. The PMCHWT-SIE formulation makes use of the equivalence principle to represent volumes of the NPs and substrate in terms of equivalent electric and magnetic currents introduced on the surfaces of these volumes. The scattered electric and magnetic fields are constructed in the form of a spatial convolution of the equivalent current densities and the Green functions of the unbounded media with permeabilities and permittivities of the volumes. To numerically solve the PMCHWT-SIE, first, the equivalent current densities are expanded using Rao-Wilton-Glisson (RWG) basis functions [6]. These expansions are inserted into PMCHWT-SIE and the resulting equation is Galerkin-tested. This yields a matrix system, which is solved for the unknown coefficients of the current density expansions. Once the current-density expansion coefficients are known, the scattered electric and magnetic fields are computed in the far field region using these expansions. At the final step, SCS is obtained [5] by integrating these scattered fields over the solid angle spanning a sphere or a hemisphere.

\section{NUMERICAL RESULTS}

The PMCHWT-SIE solver, which is briefly described in Section II, is used to analyze scattering from a group of nine hemispheres with varying radii. The hemispheres are located on a dielectric substrate. This configuration represents silver NPs fabricated on an indium tin-oxide (ITO) substrate using the annealing technique [7]. In the simulations, the permittivity of the silver is calculated using the Johnson-Christy model [8] and relative permittivity of the ITO is 4.2. Dimensions of the substrate are $600 \mathrm{~nm} \times 600 \mathrm{~nm} \times 30 \mathrm{~nm}$. Radii of the hemispheres change from $30 \mathrm{~nm}$ to $90 \mathrm{~nm}$. The setup is excited by a plane wave normally incident on the substrate (from $+\mathrm{z}$ 
direction to $-\mathrm{z}$ direction). Fig. 1 shows the mesh discretizing the hemispheres and the substrate from top.

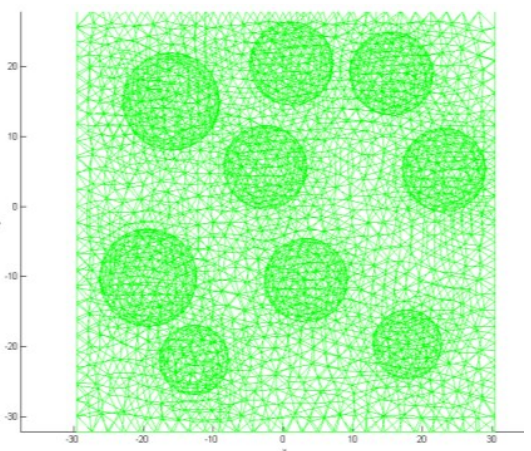

Fig. 1. Top view of the mesh discretizing the silver NPs and substrate.

This specific simulation model is inspired by the experimental setup, as described in [9], where reflection spectrum of the NPs is measured. In the simulations, as a measure of reflection, SCS is computed over the solid angle with theta spanning from $0^{0}$ to $90^{\circ}$ (hemisphere "sitting" at the top of the substrate). This is termed SCS-R. Similarly, as a measure of transmittance, SCS is computed over the solid angle with theta spanning from $90^{\circ}$ to $180^{\circ}$ (hemisphere "sitting" at the bottom of the substrate). This is termed SCS-T.

Fig. 2 plots SCS-R, SCS-T, and ACS of the setup described above versus the wavelength. SCS-T and SCS-R are very close to each other between $550 \mathrm{~nm}$ and $1200 \mathrm{~nm}$. At lower wavelengths, they differ significantly. Overall ACS is lower than SCS-R and SCS-T and it even gets lower at wavelengths higher than $700 \mathrm{~nm}$.

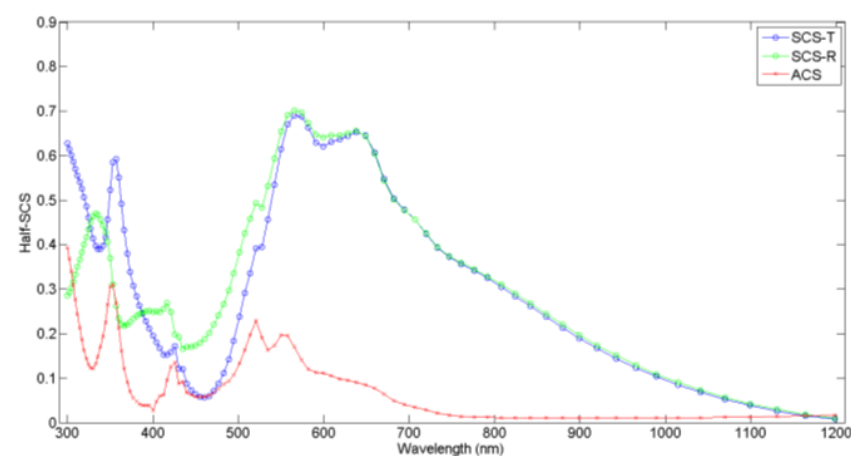

Fig. 2. SCS-R, SCS-T, and ACS computed by the PMCHWT-SIE solver for the setup with nine NPs.

The measured reflection of the silver NPs on ITO is provided in Fig. 2 in [9]. It has a single peak around $600 \mathrm{~nm}$. Note that the experimental data is between 400 and $1000 \mathrm{~nm}$. It is similar to SCS-R provided in Fig. 2, yet SCS-R has two very close peaks around $600 \mathrm{~nm}$ instead of a single and wider peak. This discrepancy might be due to the fact that the setup used by the PMCHWT-SIE solver does not incorporate the exact shape and size of NPs used in the experiments. Nevertheless, SCS-R of this setup is significantly closer to the experimental results than SCS-R of a single sphere or a hemisphere residing in vacuum as shown in Fig. 3.

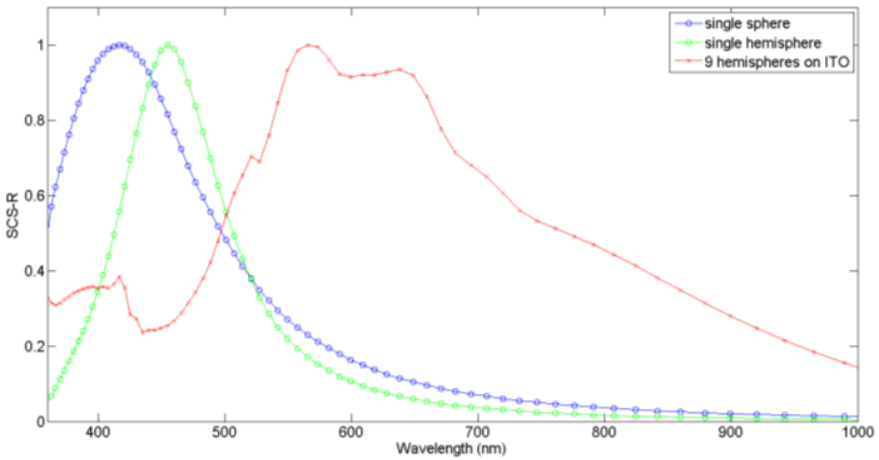

Fig. 3. SCS-R computed by the PMCHWT-SIE solver for the setup with nine NPs, a sphere in vacuum, and a hemisphere in vacuum.

\section{CONCLUSIONS}

Carefully designed and fabricated NPs have the potential to boost up the efficiency of a solar cell by directing more light energy to the active region of the cell. This means that for increased efficiency, when NPs are placed on top of the cell, their SCS-T has to be increased while their SCS-R and ACS have to be decreased.

In this work, an accurate analysis of scattering from the silver NPs fabricated on an ITO substrate is carried out using a PMCHWT-SIE solver. Computed SCS-R is in good comparison with the measured reflection of the NPs as described in [9]. This clearly shows that electromagnetic interactions between the substrate and multiple particles have to be taken into account to obtain accurate results.

\section{ACKNOWLEDGMENT}

This work is partially supported by the Scientific and Technical Research Council of Turkey (TUBITAK) under the Grant No. 115F099. The authors I. E. Uysal and O. Gulseren would like to thank TUBITAK-ARDEB for their support.

\section{REFERENCES}

[1] P. Mandal and S. Sharma, "Progress in plasmonic solar cell efficiency improvement: A status review," Renewable and Sustainable Energy Reviews, vol. 65, pp. 537-552, 2016.

[2] H. A. Atwater and A. Polman, "Plasmonics for improved photovoltaic devices," Nat. Mater., vol. 9, no. 3, pp. 205-213, 2010

[3] D. C. Tzarouchis, P. Ylä-Oijala, T. Ala-Nissila, and A. Sihvol, "Shape effects on surface plasmons in spherical, cubic, and rod-shaped silver nanoparticles," Appl. Phys. A, 122:298, 2016.

[4] L. N. Medgyesi-Mitschang, J. M. Putnam, and M. B. Gedera, "Generalized method of moments for three-dimensional penetrable scatterers," JOSA A, vol. 11, no. 4, pp. 1383-1398, 1994.

[5] I. E. Uysal, H. A. Ulku, and H. Bagci, "Transient analysis of electromagnetic wave interactions on plasmonic nanostructures using a surface integral equation solver," J. Opt. Soc. Am. A, vol. 33, pp. 17471759, 2016.

[6] S. Rao, D.Wilton, and A. Glisson, "Electromagnetic scattering by surfaces of arbitrary shape," IEEE Trans. Antennas Propag., vol. 30, pp. 409-418, 1982.

[7] P. Albella, et. al., "Shape matters: Plasmonic nanoparticle shape enhances interaction with dielectric substrate," Nano Lett., vol. 11, pp. 3531-3537, 2011.

[8] P. B. Johnson and R. W. Christy, "Optical constants of the noble metals," Phys. Rev. B, vol. 6, pp. 4370-4379, 1972.

[9] I.Tanyeli, H. Nasser, F. Es, A. Bek, and R. Turan, "Effect of surface type on structural and optical properties of Ag nanoparticles formed by dewetting," Opt. Express, vol. 21, pp. A798-A807, 2013. 